\title{
Vector Vortex Coronagraph: first results in the visible
}

\author{
Dimitri P. Mawet ${ }^{a}$, John T. Trauger ${ }^{a}$, Eugene $\operatorname{Serabyn}^{a}$, Dwight C. Moody ${ }^{a}$, Kurt M. Liewer ${ }^{a}$, \\ John E. Krist ${ }^{a}$, David M. Shemo ${ }^{b}$, Nada A. O’Brien ${ }^{b}$ \\ ${ }^{a}$ Jet Propulsion Laboratory, California Institute of Technology, 4800 Oak Grove Drive, \\ Pasadena, CA 91109, USA \\ ${ }^{b}$ JDSU, 2789 Northpoint Parkway, Santa Rosa, CA 95407, USA
}

\begin{abstract}
We report the status of JPL and JDSU ongoing technological developments and contrast results of the vector vortex coronagraph (VVC) made out of liquid crystal polymers (LCP). The first topological charge 4 VVC was tested on the high contrast imaging testbed (HCIT) around $800 \mathrm{~nm}$, under vacuum and with active wavefront control (32x32 Xinetics deformable mirror). We measured the inner working angle or IWA (50\% off-axis transmission) at $\sim 1.8 \lambda / d$. A one-sided dark hole ranging from $3 \lambda / d$ to $10 \lambda / d$ was created in polarized light, showing a mean contrast of $\sim 2 \times 10^{-7}$ over a $10 \%$ bandwidth. This contrast was maintained very close in $(3 \lambda / d)$ in a reduced $2 \%$ bandwidth. These tests begin to demonstrate the potential of the LCP technology in the most demanding application of a space-based telescope dedicated to extrasolar planet characterization. The main limitations were identified as coming from incoherent sources such as multiple reflections, and residual chromaticity. A second generation of improved masks tackling these issues is being manufactured and will be tested on the HCIT in the coming months.
\end{abstract}

Keywords: High contrast imaging, exoplanets, phase-mask coronagraphy, optical vortex, polarization manipulation, wavefront control

\section{INTRODUCTION}

Direct imaging of exoplanets is very challenging, not only because of the small angular separation between planets and stars (e.g. $<500$ mas for a 5 -AU distance at $10 \mathrm{pc}$ ), but even more because in the optical regime the contrast between a planet and its host star ranges from $10^{-3}$ for very close hot giant planets to $10^{-10}$ for Earth-like planets. Nevertheless, direct imaging is a most promising method as it provides a straightforward means to characterise planetary atmospheres with spectro-photometric measurements. Coronagraphy, a means of suppressing bright starlight so as to search for faint companions or circumstellar material, has garnered much interest in the past decade. A number of new optimized coronagraph concepts has been considered for dedicated exoplanet-finding projects at both ground- and space-based observatories (see the rather exhaustive survey of Guyon et al. ${ }^{1}$ ). Four groups of coronagraphs can be identified. In the pupil plane coronagraph family, there are: (a) amplitude apodization (via either intensity modification, or pupil shaping), and the phase induced amplitude apodization (PIAA) approach, in which the mirrors are warped to reshape the distribution of light in the pupil to yield an apodized shape, and (b) pupil plane phase apodization. In the focal plane coronagraph family, there are: (c) amplitude masks (classical Lyot coronagraphs and band-limited profiles), and (d) phase-mask coronagraphs of various types. Unlike "Lyot family" amplitude coronagraphs, focal-plane phase masks such as the four-quadrant phase-mask (FQPM, see Refs. 2 and 3 for a recent review on the FQPM) and optical vortex coronagraphs ${ }^{4-7}$ have the great advantage of enabling a very small IWA without trading off throughput.

Further author information: (Send correspondence to Dimitri Mawet)

E-mail: Dimitri.Mawet@jpl.nasa.gov, Telephone: 18183540675

Techniques and Instrumentation for Detection of Exoplanets IV, edited by Stuart B. Shaklan, Proc. of SPIE Vol. 7440, 74400X - (C) 2009 SPIE · CCC code: 0277-786X/09/\$18 · doi: 10.1117/12.826569 


\section{OPTICAL VECTORIAL VORTEX CORONAGRAPH}

An optical vortex is a phase singularity in an optical field, a point of zero intensity, resulting from a phase screw dislocation of the form $e^{i l \theta}$, with $l$ being the so-called topological charge*, and $\theta$ the azimuthal coordinate. This anomaly forces the intensity to vanish by a total destructive interference, creating a dark core. This dark core propagates and is conserved along the optical axis. ${ }^{8,9}$ Whether a dark core is created in the pupil or focal plane of a telescope will determine the way it further evolves. In Ref. 4, we analytically and numerically demonstrated that the creation of a vortex of any even topological charge $l$ at the focal plane can lead to a perfect coronagraph in the ideal case (no further phase aberrations in the system). This result has also been confirmed. ${ }^{5,6}$

Comparing coronagraphs for exoplanet detection, Guyon et al. ${ }^{1}$ identified two as closest to ideal: the phaseinduced amplitude apodization (PIAA) coronagraph, and the optical vortex phase-mask coronagraph. ${ }^{4}$ The optical vortex coronagraph is ideal in several ways: it benefits from a small IWA, and provides a very high throughput (more than 90\%). We note that because of their small IWA, and lack of "features" anywhere on the mask (except at the central singularity), the discovery space of the vortex phase-mask coronagraphs is close to maximal in terms of search area for faint companions (no dead zones).

There are two kinds of optical vortices (and thus of elements that induce them):

- the scalar optical vortex, based on the longitudinal phase delay (which applies to both polarizations identically), and is implemented by a structural helical phase ramp; ${ }^{5,7}$

- the vectorial optical vortex, based on the "geometrical" or Pancharatnam phase, ${ }^{10}$ obtained by manipulating the transverse polarization state of the light with space-variant birefringent optical elements (SVBOE).

\subsection{Principle of the vector vortex}

A vector vortex, like a scalar vortex, presents the same screw phase dislocation $e^{i(l \theta)}$ that induces the central phase singularity. A vector vortex or more rigorously an optical vectorial vortex is a SVBOE with rotational symmetry properties. It is a halfwave plate in which the optical axes rotate about the center (Fig. 1). The mathematical development based on the Jones matrix formalism and the Pancharatnam-Berry or geometrical phase is presented in Refs. 4 and 11. Note that the final result of this development is the so-called imperfect VVC "canonical formula" in the circular polarization basis, given in terms of Jones matrices as,

$$
J_{v}(r, \theta)=V\left[\begin{array}{cc}
0 & e^{i l_{p} \theta} \\
e^{-i l_{p} \theta} & 0
\end{array}\right]+L\left[\begin{array}{ll}
1 & 0 \\
0 & 1
\end{array}\right]
$$

where $l_{p}$ is the vector vortex topological charge (defined on the Pancharatnam geometrical phase in lieu of the longitudinal phase), $r$ and $\theta$ the polar azimuthal and radial coordinates, respectively. Note that $J_{v}$ is composed of two main terms. The first term is the pure vortex term of weight $V$, bearing the geometrical phase ramp structure $e^{i l_{p} \theta}$. This phase modification is geometrical, i.e. induced only by the space variation of the optical axis orientation across the component. Subsequently, it is achromatic by nature. The second term with a weighing coefficient $L$ bears no phase modification and is a leakage term. A very important property of vectorial vortices versus scalar vortices, is that the polarization structure of the chromatic leakage due to the local imperfections of the HWP is orthogonal to the pure achromatic vortex term: note the different directions of the two Jones matrices of $J_{v}$ for circular polarization. This important fundamental difference between the two approaches suggests that one might be able to reduce the effect of the manufacturing defects (retardance and transmittance mismatches) by appropriate polarization filtering (Mawet et al., in preparation).

${ }^{*}$ The topological charge of a vortex is defined according to the number of $2 \pi$ radians that the phase of the scalar wave accumulates along a closed path surrounding the phase singularity

$$
l=\frac{1}{2 \pi} \oint \nabla \phi d s
$$

where the integration path encircles the central singularity. 


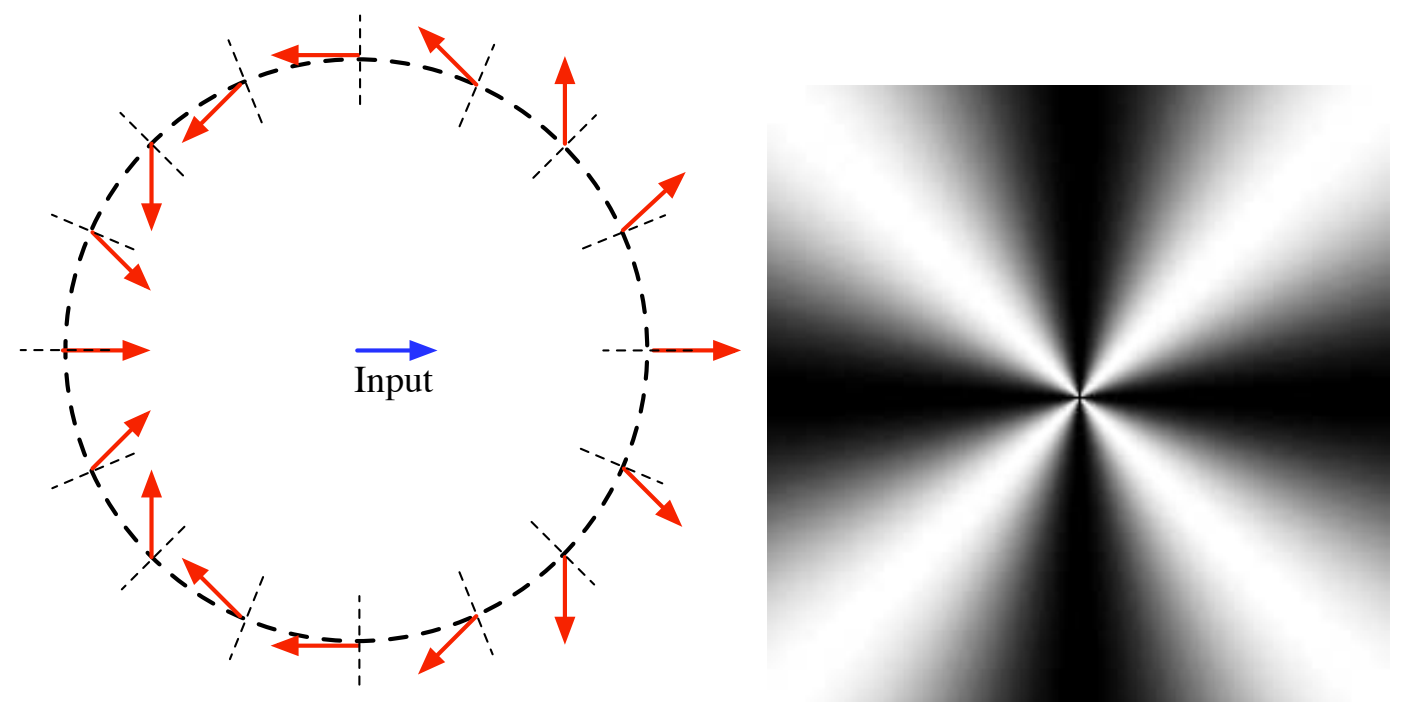

Figure 1. Particular example of a VVC of topological charge $l_{p}=2$, illustrating the concepts needed to describe vectorial vortices. Left: the figure shows a HWP with an optical axis orientation $\omega$ that rotates about the center as $\omega=\theta$, where $\theta$ is the azimuthal coordinate (dashed lines perpendicular to the circumference). The textbook net effect of a HWP on a linear impinging polarization is to rotate it by $-2 \times \alpha$ where $\alpha$ is the angle between the incoming polarization direction and the extraordinary $(e)$ optical axis . Applying this property to the space-variant HWP, assuming an incoming horizontal linear polarization, we see that the latter is transformed by the vectorial vortex so that it spins around its center twice as fast as the azimuthal coordinate $\theta$ (arrows). In this case, the angle of local rotation of the polarization vector $\phi_{p}$ corresponds to the "geometrical" or Pancharatnam phase: upon a complete rotation about the center, $\phi_{p}$ has undergone a total $2 \times 2 \pi$ virtual phase ramp, which corresponds to the definition of an optical vortex of topological charge 2 . Right: computation of the periodic modulation of the intensity transmitted by an VVC between crossed polarizers.

\subsection{LCP technology}

LCPs are materials that combine the birefringent properties of liquid crystals with the excellent mechanical properties of polymers. The orientation of a LCP is achieved through photo-alignement. Once aligned and cured, the polymer reaches a very stable solid state. The detailed proprietary fabrication process of our manufacturer JDSU has already been described in the proof of concept paper that tested the very first sample. ${ }^{11}$ In that previous paper, we obtained honorable and encouraging results, experimentally validating the VVC concept. However, this very first near-infrared (H-band) sample was limited by the size of the so-called region of disorientation $(\sim 70$ microns in diameter). This defect, centered on the vortex singularity was due to the LCP arbitrary orientation at the very center of the sample where most of the energy of the Airy diffraction pattern of the central point source (a simulated star) effectively lands.

Next generation samples were improved by significantly shrinking the central region of disorientation and covering it by an well-sized opaque Aluminum mask. Numerical simulations presented in Ref. 11 promised dramatic improvements in contrast if those prescriptions were to be respected. The shrinking was to be made possible by a redesign of the manufacturing apparatus described in Ref. 11.

\section{VVC4: VECTOR VORTEX CORONAGRAPH OF TOPOLOGICAL CHARGE 4}

For a medium size telescope (1-2 meters), the topological charge 4 represents the best compromise between IWA and a manageable sensitivity to low-order aberrations. Indeed, the theoretical IWA of a topological charge 4 VVC is $1.75 \lambda / d$ (Fig. 2), corresponding to 75 mas for a 1.5-meter telescope at a wavelength of $550 \mathrm{~nm}$. In other words, it could allow high contrast imaging of off-axis sources inside a radius of 1 AU for a system situated at $10 \mathrm{pc}$ with a probe-class space telescope operating in the visible.

In addition, the optical vortex coronagraph attenuation sensitivity to low-order aberrations like tip-tilt $t$ (pointing error) has been shown, numerically in Refs. 4 and 1, and analytically in Ref. 6 to be proportional to 

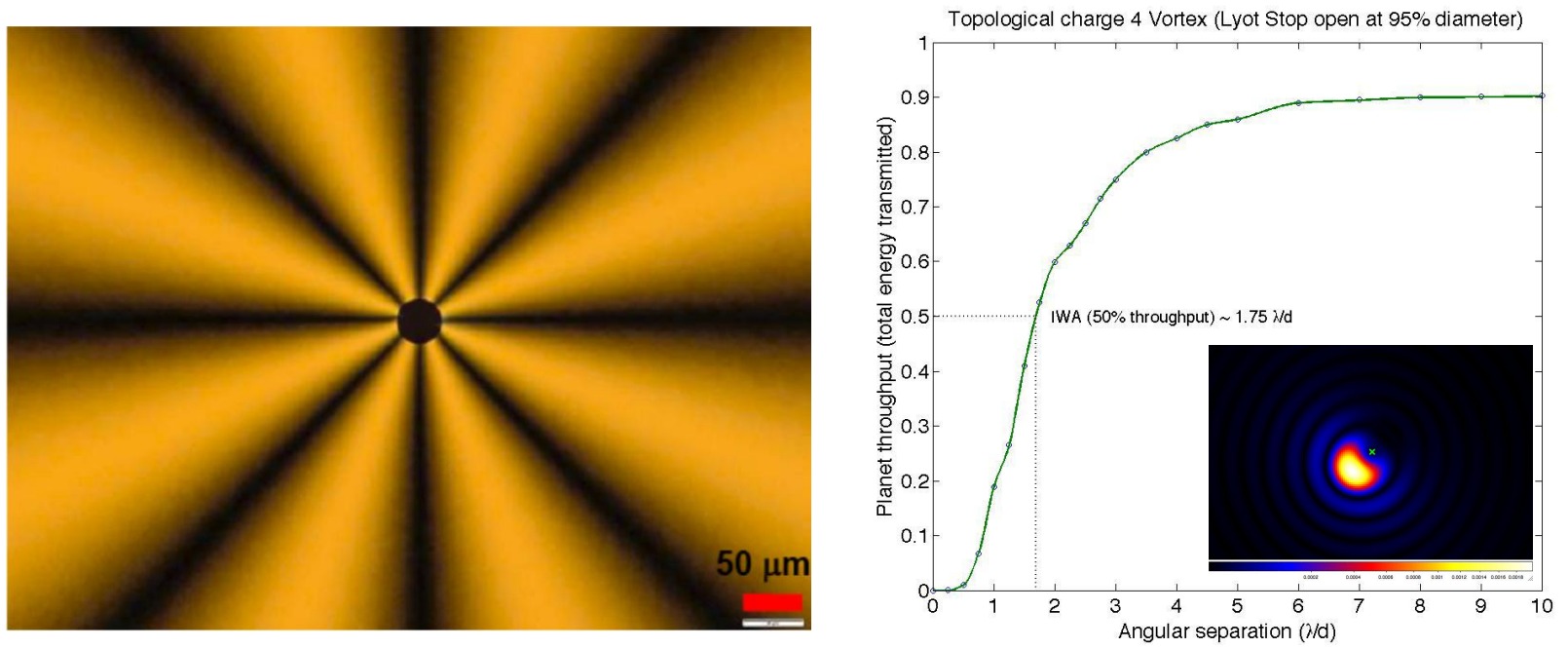

Figure 2. Left: topological charge 4 optical VVC sample between crossed polarizers after the lamination of the cover substrate on which the $\mathrm{Al}$ spot is coated (diameter of 40 microns). Note the good centering of the spot on the vortex center and the almost perfect symmetry of the pattern, which contains twice more azimuthal modulations than the topological charge 2 samples. Right: theoretical curve of the off-axis transmission allowing to define the $50 \%$ off-axis throughput IWA at $1.75 \lambda / d$. The insert shows the shape of the off-axis point-spread function (PSF) at the IWA.

$t^{l}, l$ being the topological charge of the vortex. For a topological charge 4 vortex, a 0.5 -mas pointing error on a 1.5 -meter telescope at a wavelength of $550 \mathrm{~nm}$ leads to a mere $\sim 10^{-11}$ degradation of the contrast at $2 \lambda / d$.

Leakage due to the finite size of stars can also be very easily estimated using a model of a uniform stellar disk which is a superposition of incoherent point sources. Assuming for instance an angular size of $0.01 \lambda / d(\lambda$ is the observation central wavelength and $d$, the telescope diameter), one gets a contrast leakage at $2 \lambda / d$ of $5 \times 10^{-7}$ for a topological charge $l=2,5 \times 10^{-11}$ for $l=4,5 \times 10^{-15}$ for $l=6$, respectively.

However, the manufacturing process for higher topological charges degrades the size of the region of disorientation. For the most recent generation of masks, we obtained a region of disorientation of 16 microns in diameter for a topological charge 2, and 36 microns in diameter for a topological charge 4 . Twofold to fourfold improvements are expected for the upcoming new generation.

\subsection{Microscope polarimetry}

The polarization properties of our first prototype were mapped on an Axoscan Mueller Matrix Spectro-Polarimeter available from Axometrics. The sample was placed on an $\mathrm{x}-\mathrm{y}$ stage and mapped at a $0.5 \mathrm{x} 0.5 \mathrm{~mm}$ xy-resolution over a 20x20 mm area. Those measurements allowed us to extrapolate the central wavelength (where the retardance is $\pi$ radians) of $788.5 \mathrm{~nm}$, very close to the specified $784 \mathrm{~nm}(\sim 0.5 \%$ accuracy). The pattern symmetry as shown in Fig. 2 is almost perfect. The Al spot centering is well matched to the modulation pattern and covers the central region correctly.

\subsection{Expected results with a perfect static wavefront}

We ran numerical simulation using PROPER ${ }^{12}$ to assess the potential of this first optical sample, given the size of the residual central region of disorientation and the fact that the retardance dispersion of a single layer of LCP is known to be chromatic. The polarimetric measurements allowed us to extrapolate the central wavelength of the mask. Knowing the birefringence dispersion of the LCP, it is then easy to derive the standard deviation of the retardance over the test wavelength range, $\sigma$.

We then assumed a perfect wavefront at a single wavelength $(788.5 \mathrm{~nm})$ and the sources of defect mentioned above. The results in terms of contrast were $10^{-6}$ at $3 \lambda / d$. The main source of monochromatic contrast degradation with a perfect wavefront is the size of the $\mathrm{Al}$ spot covering the central region of disorientation, which creates diffraction effects, causing light to leak from the edges in the Lyot plane. 

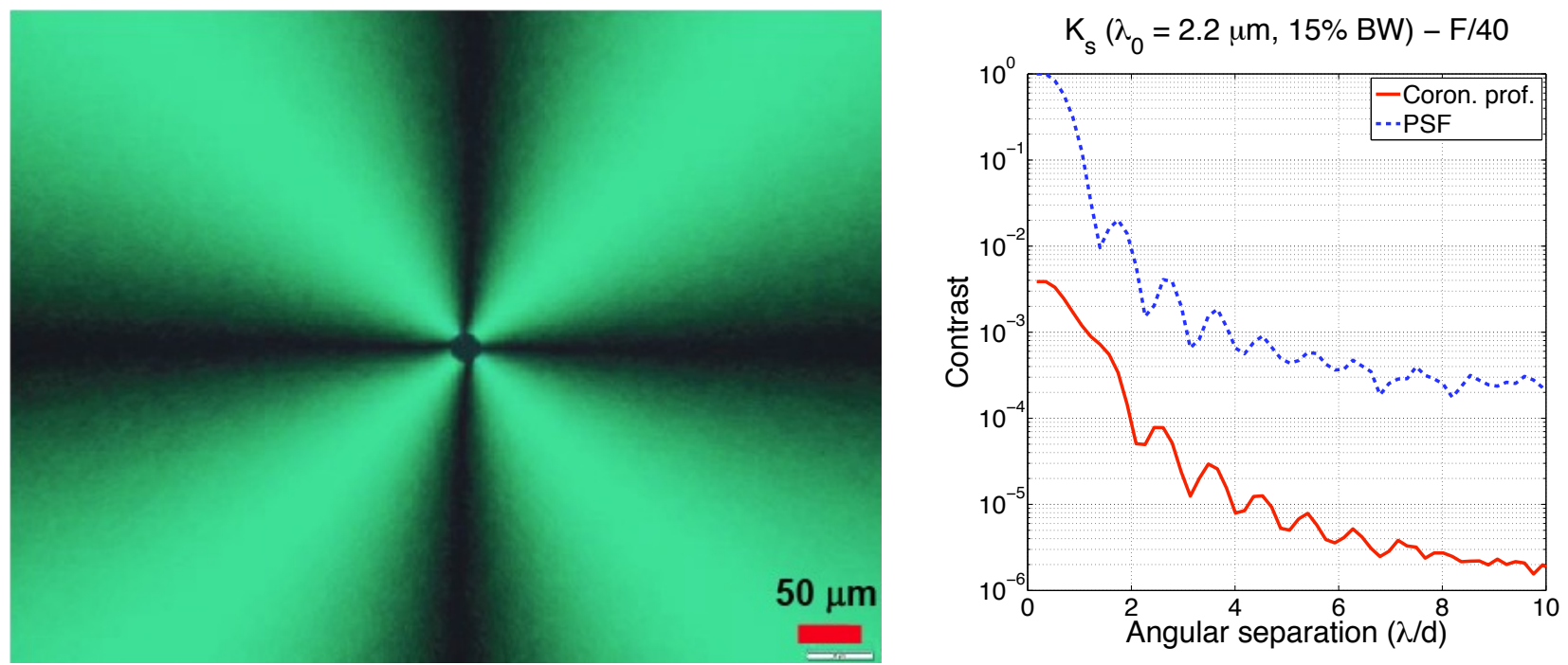

Figure 3. Left: topological charge $2 \mathrm{~K}$-band VVC sample between crossed polarizers after the lamination of the cover substrate on which the $\mathrm{Al}$ spot is coated (diameter of 25 microns). Note the good centering of the spot on the vortex center and the almost perfect symmetry of the pattern. Right: coronagraphic profiles measured in the $\mathrm{K}_{s}$ band (around $\lambda=2.2 \mu \mathrm{m}$, with a $15 \%$ bandwidth). The contrast at the $3 \lambda / d$ angular separation is $\sim 10^{-5}$ for a $15 \%$ bandwidth.

\section{OPTICAL TESTS ON THE HCIT}

Here we present our most recent laboratory results in the visible on the HCIT with the first VVC4. Our goal is to bring the technology to performance and maturity levels sufficient for the most demanding application of a space-based telescope dedicated to extrasolar planet imaging and characterization. Note that we are also pursuing developments of VVCs in the near-infrared, where we recently demonstrated performance sufficient to consider this technology for implementation in coronagraphic instruments behind current-generation adaptive optics systems (Fig. 3; Mawet et al., in preparation), and even behind next-generation extreme adaptive optics (ExAO) such as SPHERE [13, Spectro-Polarimetric High-contrast Exoplanet Research], GPI [14, Gemini Planet Imager], Palm-3000 [15, High-Order Adaptive Optics Upgrade for Palomar Observatory], HiCIAO [16, High Contrast Imaging Adaptive Optics for Subaru].

\subsection{Experimental setup}

The HCIT, a laboratory facility at JPL, is the state-of-the-art coronagraphic bench (see Ref. 17 for details). Using a reflective scheme, this facility highlights a vacuum chamber mounted on vibration isolation blocks, and a high-order 32x32 deformable mirror (DM) which is the key to the necessary wavefront active control.

The HCIT light source is a supercontinuum laser, filtered by one of 6 passband filters; 5 filters have center wavelengths of $768,784,800,816$, and $832 \mathrm{~nm}$ and (nearly square) $16 \mathrm{~nm}$ bandpasses $(\Delta \lambda / \lambda=2 \%)$. Inside the tank, the light from the source is passed through an unresolved pinhole $(\mathrm{d}=5 \mu \mathrm{m}$, compared to $\mathrm{F} \lambda=20 \mu \mathrm{m})$, effectively acting as a point-source.

When power is applied to the DM, the phase errors of the system are dominated (at the spatial frequencies of interest to us) by the surface of the DM, whose actuators have differing voltage-to-displacement relationships. After powering up the DM, the first activity is to flatten the system, using a modified Gerchberg-Saxton estimation procedure. The goal of this initialization step is to produce a front-end exit pupil with a uniform phase across the aperture, where front-end refers to the optics preceding the occulter. This technique typically leaves a $5 \mathrm{~nm}$ rms residual front-end wavefront error.

At periodic intervals during normal operation, the occulter is re-centered on the image of the source. Remotely controlled actuators translate the occulting mask to compensate slow environmental drifts. The photometric calibration is also periodically recalculated. The photometric calibration scheme used relates images taken with the occulter in-place to images taken with the occulter out-of-place, establishing a ratio between the light at 

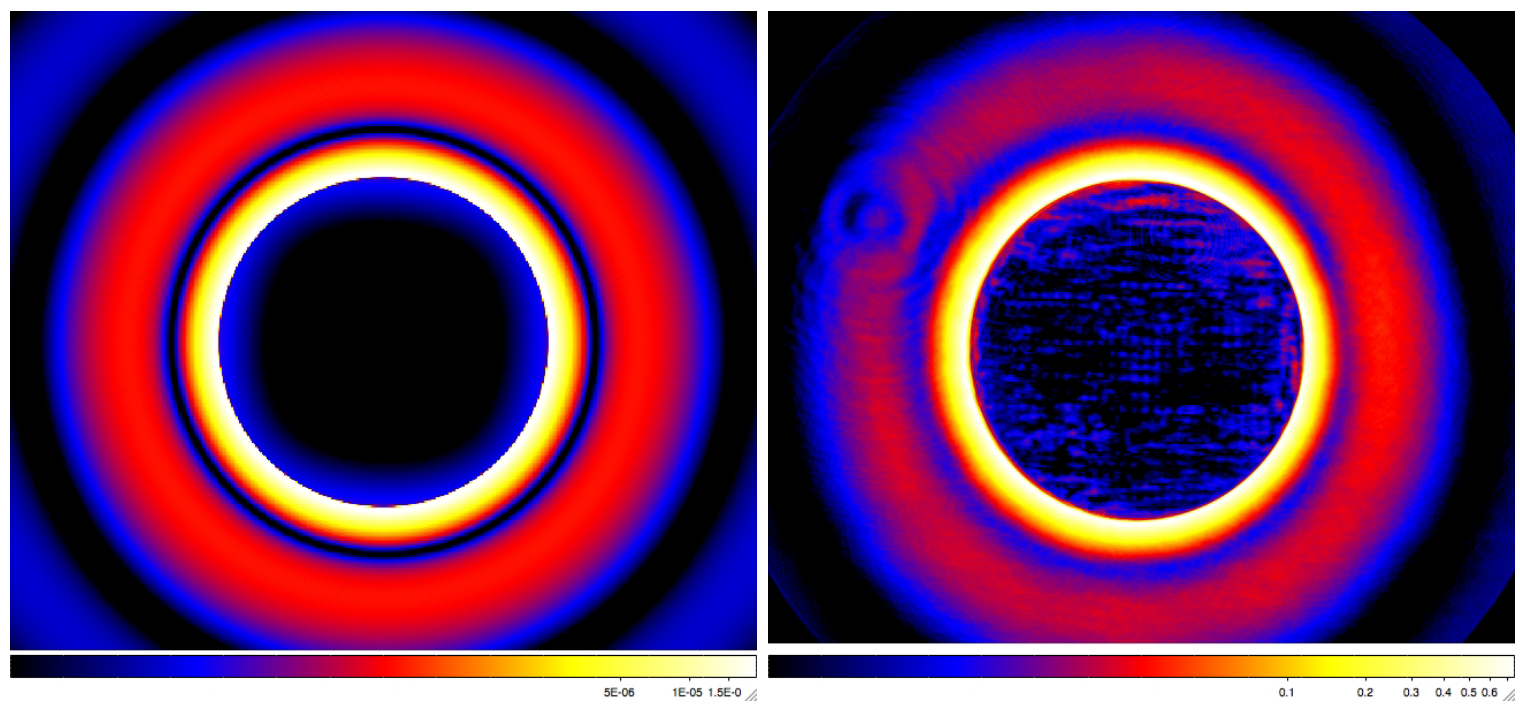

Figure 4. Pupil images from the model (left) and actual measurement on the HCIT using its pupil imaging mode (right). Note the very sharp edge defining the pupil contour. The scale is logarithmic.

the peak of the unocculted PSF to the wings of the occulted PSF (well beyond the region in the image directly controllable to the Nyquist spatial frequency of DM actuators). This ratio has been seen empirically to be quite stable (few \% variations over months), and is used to calibrate photometrically every image taken during normal operation.

To create a so-called dark hole, i.e. a region of the image were the wavefront correction provided by the $\mathrm{DM}$ is applied, we used the electric field conjugation algorithm [18, EFC]. The name of the algorithm comes from the fact that the DMs actuators are set so as to superpose the negative of the electric field onto the image plane, conceptually making the image intensity zero. The algorithm is divided into two parts, estimation and correction. The complex electric field amplitude in the image plane is estimated, then the estimate is processed to determine a correction to be applied to the DM actuators. These two parts are functionally independent, and each could be used separately with other approaches. Each EFC iteration begins with a DM setting which is the result of the prior iterations correction result (or is the flat setting, to begin the first iteration).

\subsection{Pupil imaging}

The HCIT is equipped with a pupil imaging mode, that is simply a re-imaging lens that can be inserted in the optical path. This mode allows us to check the consistency of our model to the actual behavior of the VVC in the pupil plane. Indeed, the action of the VVC in the pupil is dramatic, rejecting all the light originally inside the pupil, outside of the latter in an axially symmetric fashion (Fig. 4). The agreement between the model and the measurement is outstanding as shown in Fig. 4.

\subsection{Measured IWA}

The effective IWA of the VVC was measured on the bench to be $\sim 1.8 \lambda / d$, which corresponds to the theoretical value for a topological charge 4 vortex (see Fig. 2, right).

\subsection{Measured Contrast Results}

After trying different sources (LASER at $784 \mathrm{~nm}$, then supercontinuum in $2 \%$ filters), different Lyot stop values $(63 \%, 80 \%)$, and wavefront sensing schemes (unpolarized, polarized), we arrived at the following configuration, providing the best results for this first prototype:

- Wavefront-control limited IWA set at $3 \lambda / d$.

- Lyot stop open at $80 \%$ diameter. 

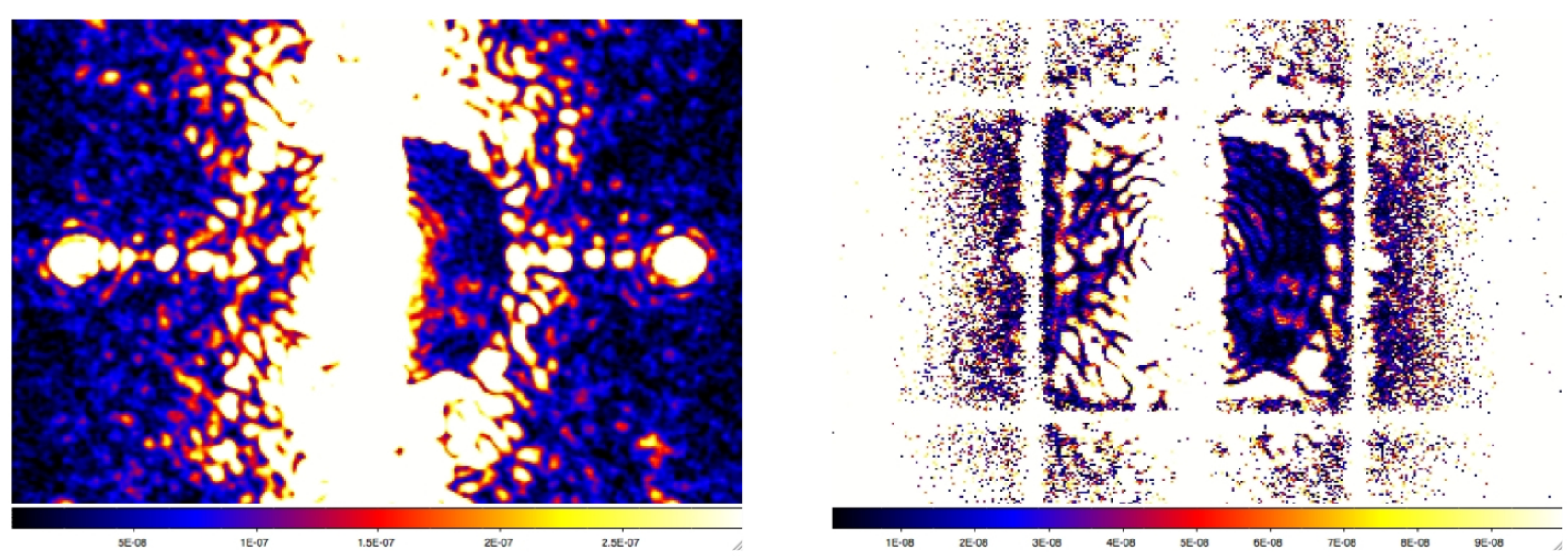

Figure 5. HCIT dark hole. Left: direct image (incoherent). Right: reconstructed image calculated from the electric field retrieved from the phase diversity applied by the DM in the wavefront sensing procedure (coherent).

- Sensing at $800 \mathrm{~nm}$, and measuring in $52 \%$-filters from 768 to $832 \mathrm{~nm}$.

- Circular polarizing beamsplitter in front of the camera, sensing and correcting in 1 polarization.

The result in terms of contrast at the IWA (3-4 $\lambda / d$, small box) and in the whole field is $2 \times 10^{-7}$ at $768 \mathrm{~nm}$ (see Fig. 5 and Fig. 6), which was a surprise for two reasons. First, the wavelength at which the wavefront sensing is performed, and for which the DM solution is calculated, is at $800 \mathrm{~nm}$ (see the plain curve in Fig. 6, showing a very obvious dip down to $5 \times 10^{-10}$ at that particular wavelength). Second, the sample central wavelength (where the retardance ought to be $\pi$ radians according the Axoscan polarimetric measurement) should be 788.5 $\mathrm{nm}$. We would have expected the chromatic leakage to be minimal at this wavelength. It is still unclear why contrast is best at the shortest wavelength.

\subsection{Discussion}

The important conclusions of those preliminary tests were:

- The result with wavefront control is 10 times better than expected with a perfect wavefront (see Sect. 3.2).

- Splitting the polarizations and sensing in one polarization only at the end helps the wavefront control system, and appears necessary to get to the highest contrast.

- The reconstructed image (calculated from the electric field retrieved from the phase diversity applied by the DM in the wavefront sensing procedure) shows the same speckle morphology as the direct image, but at a level which is significantly lower (the coherent contrast is at the $10^{-9}$ level, with an excursion at $5 \times 10^{-10}$ at the wavefront control wavelength).

- The gap between the reconstructed and direct image is smaller at small wavelength (Fig. 6).

- The chromaticity of the reconstructed image and direct image is different, with a very obvious dip and wings for the reconstructed image, and a smooth linear degradation with wavelength for the direct image (Fig. 6).

The discrepancy between the reconstructed image and direct image behavior can be explained by considering mutual coherence of the VVC terms, implying that the reconstructed image is known to show the coherent content of the beam, while any discrepancy with respect to the reconstructed signal ought to be from incoherent sources. Here is a list of incoherent sources of light that can all be attributed to imperfections of our first prototype: 


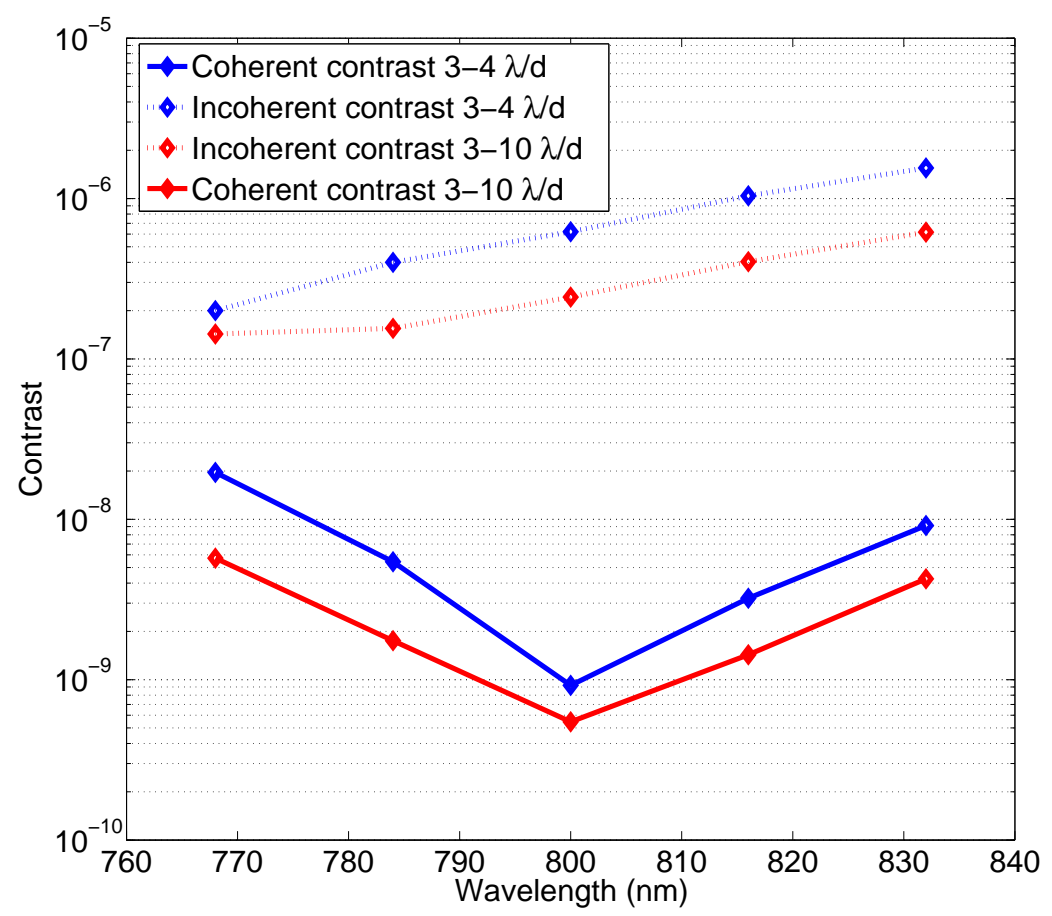

Figure 6. Contrast results obtained on the HCIT with the first topological charge 4 VVC. The blue curves show the contrast in the so-called small box $(3-4 \lambda / d, 1 \lambda / d$ square). The red ones are integrated in the whole half dark hole(3-10 $\lambda / d)$. The dotted curves show the chromatic dependence on the real measured contrast on wavelength. The plain curves show the computed contrast extracted from the reconstructed images (from the phase diversity applied to the DM).

- Ghosts originating from multiple reflections inside the multi-layer structure of the sample.

- Chromatic leakage, shown above in Equation (2) to be mutually incoherent with the pure vortex term.

Our contrast results were affected by both sources, and both will have to be worked out for the next generation of masks. We proposed the solutions to this double issue, with the superachromatic 3-layer design (Mawet et al., in preparation) that will reduce the chromatic leakage down to acceptable levels, and with a careful index matching and AR-coating of the multi-layer stack.

Note also that a full, dual polarization use of the VVC with wavefront control requires dedicated transformations of the current EFC algorithm to incorporate both polarization electric fields into the sensing and control schemes (Laurent Pueyo, Amir Give'on, personal communication). The new version of the algorithm is currently being numerically simulated, and will be experimentally attempted on the HCIT during the tests of the next generation of VVC optical samples.

\section{CONCLUSION}

We have demonstrated that a vortex synthesized out of a space-variant birefringent element, i.e. a vector vortex is an extremely powerful coronagraphic solution, as predicted. Thanks to ground-breaking advances in LCP technology, a set of viable samples was produced in a very short time. Our prototyping operation at JPL with our industrial partner JDSU quickly provided very good contrast results. Our first runs on the HCIT with an optical wavelength VVC were more than promising.

The second generation near-infrared prototypes are also compliant with the specifications of next generation ground-based instruments for exoplanet imaging and characterization, such as SPHERE,${ }^{13}$ GPI, ${ }^{14}$ Palm-3000 ${ }^{15}$ and HiCIO. ${ }^{16}$ The one-layer design, equipped with the central occulting spot, is sufficiently achromatic for direct 
implementation on the telescope for direct contrast improvements. As a matter of fact, a vectorial vortex has just been tested successfully on sky on the Palomar 5-meter Hale telescope. This will be the subject of a forthcoming paper (Mawet et al., in preparation).

The ability of LCP to synthesize optical vortices is excellent, with the potential of high-level achromatization and the straightforward possibility of implementing higher topological charges (Mawet et al., in preparation). We pursue those developments further with the goal of providing the best coronagraph for use in the context of a small space-based observatory dedicated to exoplanet imaging. ${ }^{17}$

\section{ACKNOWLEDGMENTS}

This work was carried out at the Jet Propulsion Laboratory, California Institute of Technology, under contract with the National Aeronautics and Space Administration (NASA). D. M. is supported by an appointment to the NASA Postdoctoral Program at the Jet Propul- sion Laboratory, California Institute of Technology, administered by Oak Ridge Associated Universities through a contract with NASA.

\section{REFERENCES}

[1] Guyon, O., Pluzhnik, E. A., Kuchner, M. J., Collins, B., and Ridgway, S. T., "Theoretical Limits on Extrasolar Terrestrial Planet Detection with Coronagraphs," ApJs 167, 81-99 (Nov. 2006).

[2] Rouan, D., Riaud, P., Boccaletti, A., Clénet, Y., and Labeyrie, A., "The Four-Quadrant Phase-Mask Coronagraph. I. Principle," PASP 112, 1479-1486 (Nov. 2000).

[3] Rouan, D., Baudrand, J., Boccaletti, A., Baudoz, P., Mawet, D., and Riaud, P., "The Four Quadrant Phase Mask Coronagraph and its avatars," Comptes Rendus Physique 8, 298-311 (Apr. 2007).

[4] Mawet, D., Riaud, P., Absil, O., and Surdej, J., "Annular Groove Phase Mask Coronagraph," ApJ 633, 1191-1200 (Nov. 2005).

[5] Foo, G., Palacios, D. M., and Swartzlander, Jr., G. A., "Optical vortex coronagraph," Optics Letters 30, 3308-3310 (Dec. 2005).

[6] Jenkins, C., "Optical vortex coronagraphs on ground-based telescopes," MNRAS 384, 515-524 (Feb. 2008).

[7] Swartzlander, G. A., Jr., Ford, E. L., Abdul-Malik, R. S., Close, L. M., Peters, M. A., Palacios, D. M., and Wilson, D. W., "Contrast Enhancement of Binary Star System Using an Optical Vortex Coronagraph," Optics Express 16, 10200-10207 (May 2008).

[8] Rozas, D., Law, C. T., and Swartzlander, Jr., G. A., "Propagation dynamics of optical vortices," Journal of the Optical Society of America B Optical Physics 14, 3054-3065 (Nov. 1997).

[9] Niv, A., Biener, G., Kleiner, V., and Hasman, E., "Manipulation of the Pancharatnam phase in vectorial vortices," Optics Express 14, 4208-4220 (May 2006).

[10] Berry, M., "The Adiabatic Phase and Pancharatnam's Phase for Polarized Light," Journal of Modern Optics 34, 1401-1407 (Nov. 1987).

[11] Mawet, D., Serabyn, E., Liewer, K., Hanot, C., McEldowney, S., Shemo, D., and O'Brien, N., "A laboratory demonstration of the capability to image an Earth-like extrasolar planet," Optics Express 17, 1902-1918 (Jan. 2009).

[12] Krist, J. E., "PROPER: an optical propagation library for IDL," in [Society of Photo-Optical Instrumentation Engineers (SPIE) Conference Series], Society of Photo-Optical Instrumentation Engineers (SPIE) Conference Series 6675 (Sept. 2007).

[13] Beuzit, J.-L., Feldt, M., Dohlen, K., Mouillet, D., Puget, P., Antichi, J., Baudoz, P., Boccaletti, A., Carbillet, M., Charton, J., Claudi, R., Fusco, T., Gratton, R., Henning, T., Hubin, N., Joos, F., Kasper, M., Langlois, M., Moutou, C., Pragt, J., Rabou, P., Saisse, M., Schmid, H. M., Turatto, M., Udry, S., Vakili, F., Waters, R., and Wildi, F., "SPHERE: A Planet Finder Instrument for the VLT," in [In the Spirit of Bernard Lyot: The Direct Detection of Planets and Circumstellar Disks in the 21st Century], Kalas, P., ed. (June 2007).

[14] Macintosh, B., Graham, J., Palmer, D., Doyon, R., Gavel, D., Larkin, J., Oppenheimer, B., Saddlemyer, L., Wallace, J. K., Bauman, B., Erikson, D., Poyneer, L., Sivaramakrishnan, A., Soummer, R., and Veran, J.-P., "Adaptive optics for direct detection of extrasolar planets: the Gemini Planet Imager," Comptes Rendus Physique 8, 365-373 (Apr. 2007). 
[15] Dekany, R., Bouchez, A., Britton, M., Velur, V., Troy, M., Shelton, J. C., and Roberts, J., "PALM-3000: visible light $\mathrm{AO}$ on the 5.1-meter Telescope," in [Advances in Adaptive Optics II. Edited by Ellerbroek, Brent L.; Bonaccini Calia, Domenico. Proceedings of the SPIE, Volume 6272, pp. 62720G (2006).], Presented at the Society of Photo-Optical Instrumentation Engineers (SPIE) Conference 6272 (July 2006).

[16] Tamura, M., Hodapp, K., Takami, H., Abe, L., Suto, H., Guyon, O., Jacobson, S., Kandori, R., Morino, J.-I., Murakami, N., Stahlberger, V., Suzuki, R., Tavrov, A., Yamada, H., Nishikawa, J., Ukita, N., Hashimoto, J., Izumiura, H., Hayashi, M., Nakajima, T., and Nishimura, T., "Concept and science of HiCIAO: high contrast instrument for the Subaru next generation adaptive optics," in [Ground-based and Airborne Instrumentation for Astronomy. Edited by McLean, Ian S.; Iye, Masanori. Proceedings of the SPIE, Volume 6269, pp. 62690V (2006).], Presented at the Society of Photo-Optical Instrumentation Engineers (SPIE) Conference 6269 (July 2006).

[17] Trauger, J. T. and Traub, W. A., "A laboratory demonstration of the capability to image an Earth-like extrasolar planet," Nature 446, 771-773 (Apr. 2007).

[18] Give'on, A., Kern, B., Shaklan, S., Moody, D. C., and Pueyo, L., "Broadband wavefront correction algorithm for high-contrast imaging systems," in [Society of Photo-Optical Instrumentation Engineers (SPIE) Conference Series], Society of Photo-Optical Instrumentation Engineers (SPIE) Conference Series 6691 (Sept. 2007). 